\title{
Congenital Giant Simple Symptomatic Renal Cyst in a Child - Rare Pathology - Ultrasonography and CT Evaluation - A Case Report
}

\author{
Shivesh Pandey ${ }^{1}$, Suresh Vasant Phatak², Pratik Bhansali ${ }^{3}$, Nishant Raj ${ }^{4}$, Bhavik Unadkat ${ }^{5}$ \\ 1,2,3,4,5 Department of Radiodiagnosis, Jawaharlal Nehru Medical College, \\ (DMIMS), Sawangi (Meghe), Wardha, Maharashtra, India.
}

INTRODUCTION

Simple renal cyst (SRC) is uncommon paediatric pathology. It's spherical, round to oval. Perceptible wall, anechoic, without septa, and cyst is separated from the collecting system, and the cyst has no colour Doppler flow. They may be as small as a pea or as large as a golf ball. Simple renal cysts are filled with serous fluid and have a simple epithelial covering. They are less common in children with incidences of less than 0.5 percent, while adults have incidence of more than $10 \%$, and more than 30 $\%$ in adults aged 70 and older. The exact mechanism by which renal cysts form is unknown. There are a few theories of how the disease develops. The causative factor, according to one hypothesis, is a focal ischemia in a renal tubule that causes localised obstruction. According to another hypothesis, renal cysts are caused by calyceal diverticula that have lost contact with the adjacent calyx. ${ }^{1}$

Because of the increased use of ultrasound screening (USS), more children are being diagnosed with renal cysts. Asymptomatic and symptomatic cysts (abdominal pain, hematuria, etc.) are distinguished, but mostly asymptomatic cysts develop slowly, though complications such as bleeding, infection, or rupture may occur, particularly in younger children. ${ }^{2}$

Chronic kidney disease (CKD), polycystic kidney disease, malignant transformation, and symptomatic enlargement have all been identified in paediatric patients with renal cysts. The vast majority of renal cysts, fortunately, do not develop and remain largely unchanged over time. In cases of asymptomatic simple renal cysts in infants, a conservative management approach is used. Recent research supports the use of an ultrasound-based (US) adapted Bosniak classification system to classify renal cysts in children. This would result in lower rates of surgical treatments for benign lesions, as well as less ionising radiation from needless computed tomography (CT) scans. It would also mean less extended monitoring and associated morbidity. ${ }^{3}$

\section{PRESENTATION OF CASE}

A 4-year-old girl was presented to our hospital with swelling in left lumbar and iliac region, pain, and difficulty in micturition. Child was apparently asymptomatic till the age of three months. Then parents noticed a swelling in the abdomen of child in left lumber and iliac region which was increasing in size. Child was referred for abdominal sonography.

Ultrasound sonography (USG) revealed cystic lesion of size $10.3 \times 8.3 \mathrm{~cm}$ in the lower pole of the left kidney. Pelvicalyceal system was normal. No cystic lesions were seen in other abdominal organs. On CT, left kidney showed a large exophytic cystic lesion $(10.3 \times 8.3 \times 7.6 \mathrm{~cm})$ containing clear fluid (HU 12) with thin enhancing septae that appears to be arising from and replacing the lower pole of left kidney and reaching up to the pelvis. No solid component or calcification was seen.
Corresponding Author: Dr. Suresh Vasant Phatak, Department of Radiodiagnosis, Jawaharlal Nehru Medical College (DMIMS), Sawangi (Meghe), Wardha, Maharashtra, India E-mail: suresh_phatak@yahoo.com

DOI: $10.14260 /$ jemds/2021/484

How to Cite This Article:

Pandey S, Phatak SV, Bhansali P, et al. Congenital giant simple symptomatic renal cyst in a child - rare pathology Ultrasonography and CT evaluation - a case report. J Evolution Med Dent Sci 2021;10(30):2362-2364, $10.14260 /$ jemds/2021/484

Submission 06-05-2021,

Peer Review 04-07-2021,

Acceptance 12-07-2021,

Published 26-07-2021.

Copyright (C) 2021 JEMDS. This is an open access article distributed under Creative Commons Attribution License [Attribution 4.0 International (CC BY 4.0)] 
The lesion displaced the left ureter and bowel loops towards the right side. Pelvicalyceal system was normal. These findings suggested Bosniac grade I renal cyst. Patient underwent surgery for removal of giant cyst. Intra-operative findings included. A huge cyst was seen on reaching retroperitoneum reaching up to the lateral of bladder and pushing ureter medially.

Cyst was filled with clear fluid. Cyst was seen arising from the lower pole of kidney. There was no communication with pelvicalyceal system, Sections from left renal cyst wall shows histopathological features suggestive of simple cyst. The postoperative phase went well, and the patient was discharged from the hospital after ten days

\section{DISCUSSION}

Simple cysts are usually found by chance and can be identified with cross-sectional radiological investigation. Simple renal cysts are generally incidentally found during a renal or abdominal ultrasound and can usually be diagnosed. A definitive diagnosis of renal cyst can also be made using contrast-enhanced computed tomography (CT) or magnetic resonance imaging (MRI). Ultrasound defines a simple cyst as a mass which is rounded to oval in shape which shows homogeneous anechoic material. The simple cyst must also have a sub-millimetre wall, smooth well-delimited margins, and a pronounced posterior enhancement. The cyst's boundaries should form a clear interface with the surrounding structures, and there should be perceptible wall surrounding the lesion. When all of the above conditions are fulfilled, ultrasound (USG) is nearly 100 percent reliable. 4

When a renal cyst is diagnosed, specific ultrasonographic guidelines should be used to determine if the cyst is benign. A simple cyst can be confidently identified on a non-contrast, unenhanced CT scan if the simple cyst shows density of 20 Hounsfield units or less, no central or peripheral calcification, with no septations, and has a very thin wall. A simple cyst is diagnosed on a contrast-enhanced CT scan if there is no enhancement, the simple fluid is measured, the wall is thin, and there are no calcifications. ${ }^{5}$

The Bosniak criteria helps to distinguish between nonsurgical and surgical cystic masses with the use of computed tomographic findings to direct patient management. These criteria use five different categories (I, II, IIF, III, and IV) to help radiologists, urologists, and internists convey the necessary management. A Bosniak type I cyst is a simple renal cyst. These renal cysts have a hairline-thin wall and fluid attenuation (0$20 \mathrm{HU}$ ) on CT, but no calcifications or septations, and show no enhancement. These cysts are harmless and do not need further investigation. Bosniak II cysts are simple in nature and easy to treat. Minimal calcifications or a small part of cyst shows very slightly thickened calcification in the wall or septa of the cyst. These cysts may have a few thin septa. ${ }^{6}$ This group also includes lesions of size $3 \mathrm{~cm}$ or less which shows non enhancement and have density of $>20 \mathrm{HU}$ that are not entirely intrarenal. Bosniak II cysts are considered benign and do not need to be evaluated further. ${ }^{7}$ A Bosniak IIF cyst is a cyst that needs to be imaged again to see if it is benign. If these cysts are more complex than Bosniak II cysts, but they aren't thought to be complicated enough to warrant surgery. Multiple fine septations internally with no contrast enhancement, some calcifications, and a thickening of the cyst wall can be seen in these lesions. This category includes hyper attenuating cysts which shows no contrast enhancement that are greater than three centimeters in diameter or fully intrarenal. ${ }^{8}$ A Bosniak IIF lesion did not cause locally advanced or metastatic disease. Bosniak IIF renal cysts are monitored with computed tomography or magnetic resonance imaging to see if they are becoming more complex. Depending on the complexity of the lesion, guidelines for follow-up time vary from $1-2$ years to more than 4 years. ${ }^{9}$ The walls and/or septa of a Bosniak III cyst may be dense and irregular, with detectable enhancement. Thick nodular calcifications may be present in the cyst wall and septa. These cysts have a 30 percent to 100 percent risk of being cancerous. Aside from renal malignancy, haemorrhagic cyst, renal abscess, mixed epithelial and stromal tumour, benign multi loculated cyst and cystic nephroma are among the differential diagnoses for a Bosniak III cyst. These patients typically undergo surgical resection due to the possibility of malignancy. Unless proved otherwise, if soft tissue lesion shows contrast enhancement in type Bosniak IV cyst, then it is considered malignant. Surgical resection is advised unless there are contraindications, such as patient with co morbidities or a short life expectancy
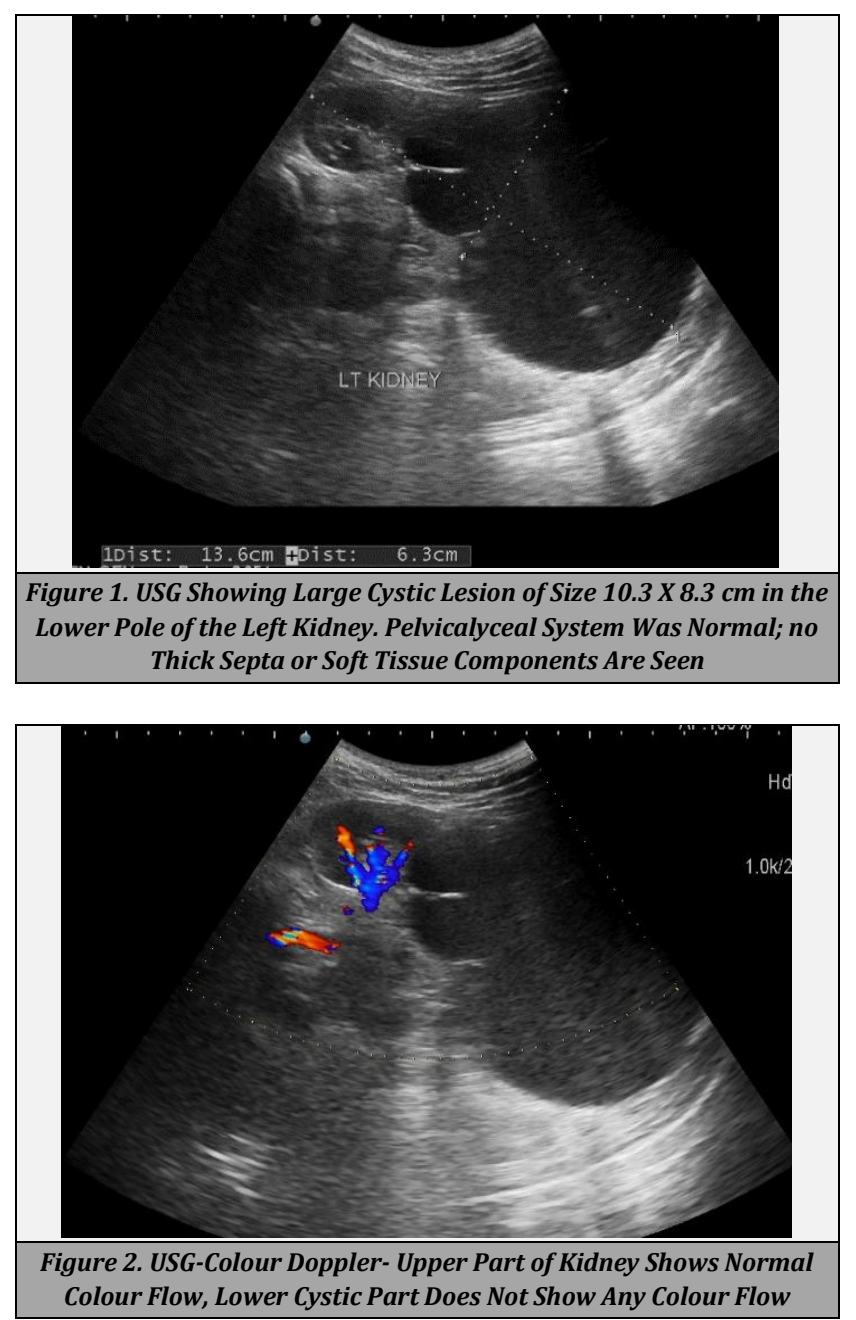

The following are the MRI characteristics of a simple cyst: on T1 weighted images showing - homogenous low signal intensity, while on T2 weighted images - homogenous high signal intensity, and no discernible contrast enhancement after gadolinium intravenous contrast. 
Contrast enhanced ultrasonography of the kidneys is a low-cost, high-resolution imaging technique. It reliably distinguishes simple cysts from indeterminate lesions, complex cysts, and infective pathology. Cystic lesions can be diagnosed using newer low mechanical index and other real time methods that use the advance generation of contrast agents. These techniques have high sensitivity for micro bubble detection and can assist with difficult cystic lesion diagnosis. Simple cysts have smooth margins, devoid of signal, and are homogenously anechoic after intravenous administration of micro bubble contrast agents. ${ }^{10}$

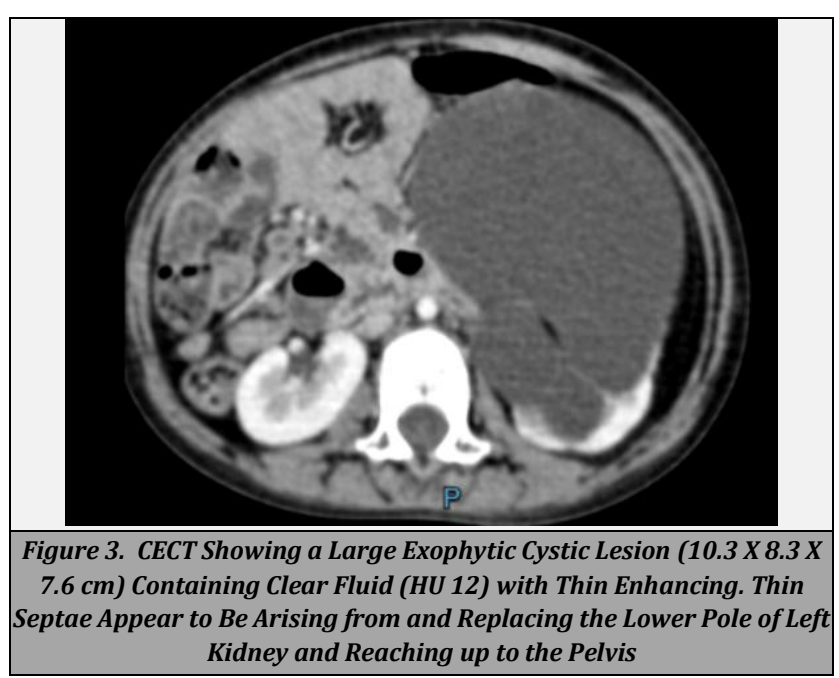

\section{DIFFERENTIAL DIAGNOSIS}

In the paediatric age group, focal renal lesions include benign congenital processes such as Calyceal diverticulum, benign mesoblastic nephroma, multilocular cystic nephroma, and lymphatic malformation.

\section{CONCLUSIONS}

Congenital simple renal cysts in children are uncommon and appear to develop slowly. However, because of the more rapid rise in cyst size in children with large cysts at diagnosis, routine radiological follow-up may be necessary. More imaging studies is required for further evaluation so as to better understand the natural history of simple renal cysts in children. For early diagnosis and better treatment of these patients, USG and CT are appropriate imaging modalities.

Financial or other competing interests: None.

Disclosure forms provided by the authors are available with the full text of this article at jemds.com.

\section{REFERENCES}

[1] Karmazyn B, Tawadros A, Delaney LR, et al. Ultrasound classification of solitary renal cysts in children. J Pediatr Urol 2015;11(3):149.e1-6.

[2] Koutlidis N, Joyeux L, Mejean N, et al. Management of simple renal cyst in children: French multicenter experience of 36 cases and review of the literature. J Pediatr Urol 2015;11(3):113-7.

[3] Bayram MT, Alaygut D, Soylu A, et al. Clinical and radiological course of simple renal cysts in children. Urology 2014;83(2):433-7.

[4] Adachi T, Nakatani T, Minami H, et al. Renal cell carcinoma with hemorrhagic cyst formation in a 4-year-old boy. Int J Urol 2003;10(5):267-70.

[5] Bosniak MA. The current radiological approach to renal cysts. Radiology 1986;158(1):1-10.

[6] Silverman SG, Israel GM, Herts BR, et al. Management of the incidental renal mass. Radiology 2008;249(1):16-31.

[7] Pooler BD, Pickhardt PJ, O'Connor SD, et al. Renal cell carcinoma: attenuation values on unenhanced CT. AJR Am J Roentgenol 2012;198(5):1115-20.

[8] Israel GM, Bosniak MA. Follow-up CT of moderately complex cystic lesions of the kidney (Bosniak category IIF). AJR Am J Roentgenol 2003;181(3):627-33.

[9] Smith AD, Remer EM, Cox KL, et al. Bosniak category IIF and III cystic renal lesions: outcomes and associations. Radiology 2012;262(1):152-60.

[10] Chu LC, Hruban RH, Horton KM, et al. Mixed epithelial and stromal tumor of the kidney: radiologic-pathologic correlation. Radiographics 2010;30(6):1541-51. 